\title{
Gradhiva
}

GRADHIV

Revue d'anthropologie et d'histoire des arts

$24 \mid 2016$

ARTchives

\section{Trente ans après : choses revues}

Entretien avec Jean Jamin

Julien Bondaz, Frédéric Keck et Jean Jamin

\section{OpenEdition}

Journals

Édition électronique

URL : http://journals.openedition.org/gradhiva/3297

DOI : $10.4000 /$ gradhiva.3297

ISSN : 1760-849X

Éditeur

Musée du quai Branly Jacques Chirac

Édition imprimée

Date de publication : 7 décembre 2016

Pagination : 206-227

ISBN : 978-2-35744-094-4

ISSN : 0764-8928

Référence électronique

Julien Bondaz, Frédéric Keck et Jean Jamin, "Trente ans après : choses revues », Gradhiva [En ligne],

24 | 2016, mis en ligne le 07 décembre 2019, consulté le 19 avril 2019. URL : http://

journals.openedition.org/gradhiva/3297 ; DOI : 10.4000/gradhiva.3297

(c) musée du quai Branly 


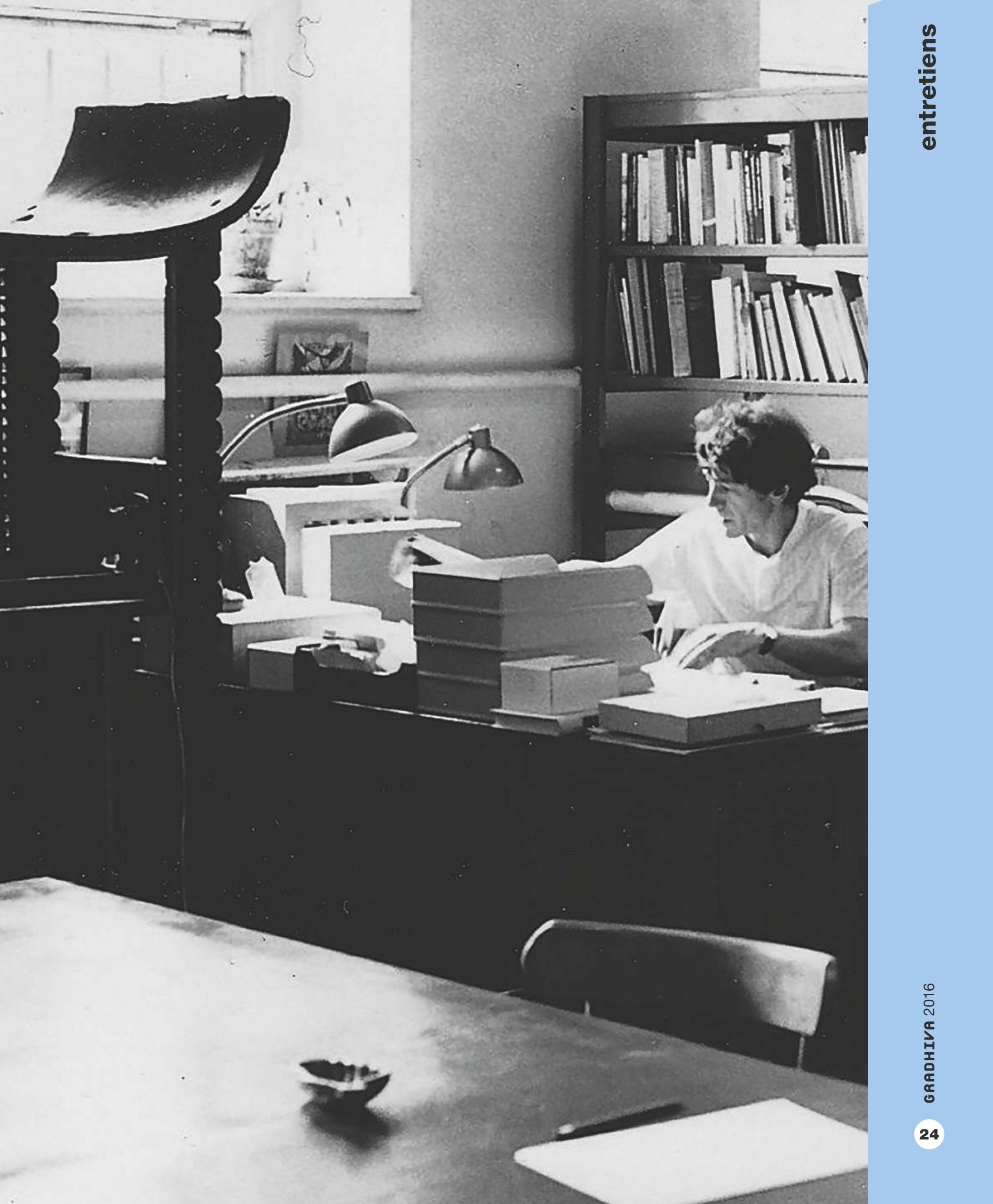




\section{Trente ans après: choses revues Entretien avec Jean Jamin ${ }^{1}$}

par Julien Bondaz et Frédéric Keck

Né en 1945 à Charleville-Mézières dans les Ardennes, Jean Jamin est directeur d'études à l'École des hautes études en sciences sociales (EHESS) où, jusqu'en 2013, il a enseigné l'épistémologie et l'histoire de l'anthropologie. Il a fondé en 1986, avec Michel Leiris, la revue Gradhiva (d'abord sous-titrée Revue d'histoire et d'archives de l'anthropologie) aujourd'hui publiée par le musée du quai Branly, avec comme sous-titre Revue d'anthropologie et d'histoire des arts. De 1997 à 2015, il a dirigé L'Homme. Revue française d'anthropologie créée par Émile Benveniste, Pierre Gourou et Claude LéviStrauss en 1961; de 2000 à 2016, il a été membre titulaire du Laboratoire d'anthropologie et d'histoire de l'institution de la culture (Lahic, Institut interdisciplinaire d'anthropologie du contemporain du CNRS, Paris), après avoir été, aux côtés de Gérard Althabe, Marc Augé, Jean Bazin, Jean-Pierre Dozon et Emmanuel Terray, un membre fondateur en 1993 du Centre d'anthropologie des mondes contemporains (CAMC) de l'EHESS. II a publié des travaux sur l'ethnologie du jazz, l'anthropologie de la littérature et l'histoire de la mission Dakar-Djibouti. Il fut récemment conseiller scientifique de l'exposition Leiris \& Co, organisée en 2015 au Centre Pompidou-Metz. Dans cet entretien, il revient sur son parcours au travers des revues d'anthropologie françaises. À un moment où celles-ci se transforment et changent de visage, Gradhiva a voulu rendre hommage à l'un de ses fondateurs et revenir avec lui sur l'itinéraire d'un homme de revues.
1. Propos recueillis à Paris le 11 janvier 2016 par Julien Bondaz et Frédéric Keck, revus en février et mars 2016 par les trois participants. 


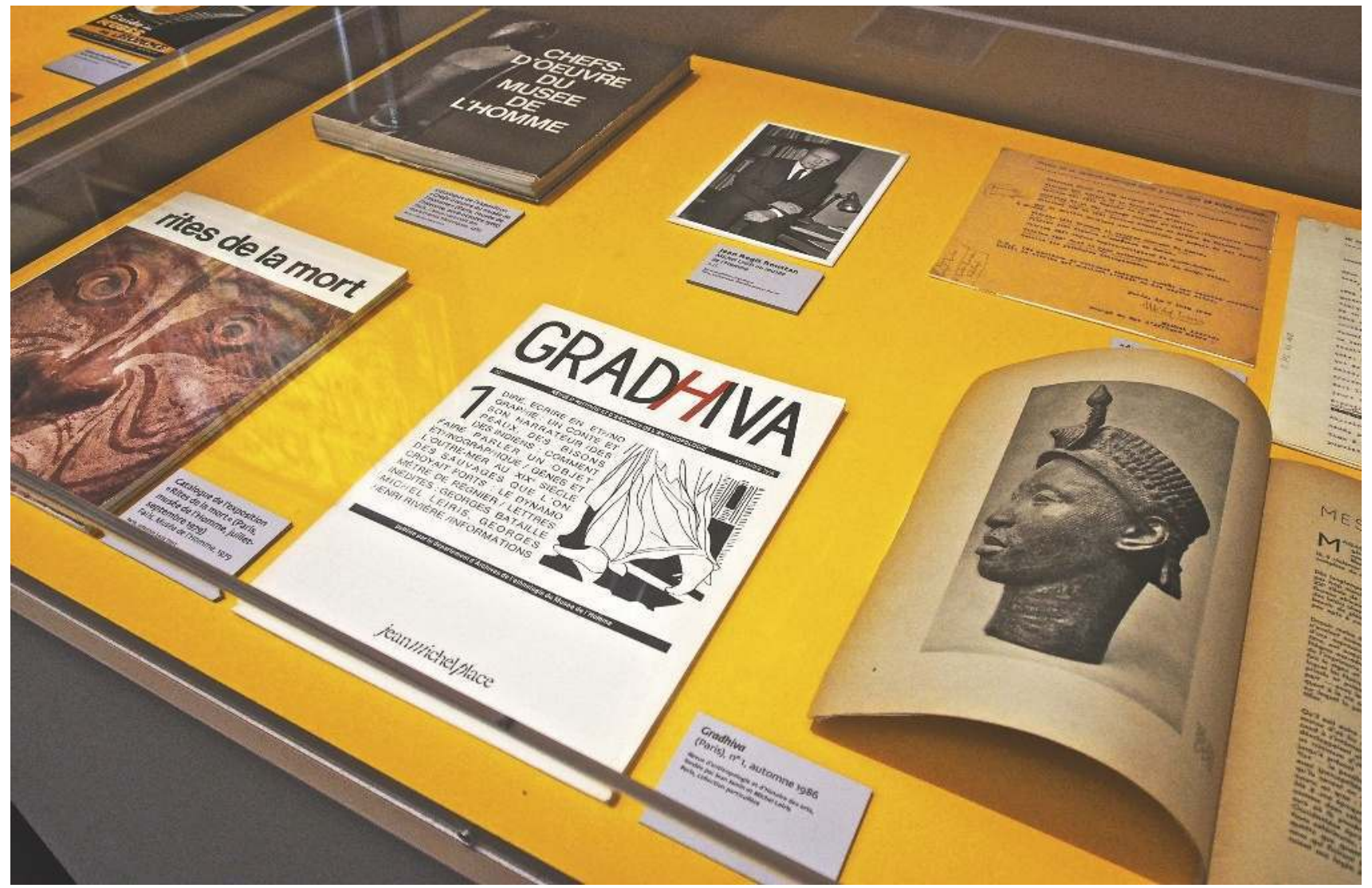

fig. 1

Gradhiva $\mathrm{n}^{\circ}$ 1, vignette de couverture dessinée par l'artiste-peintre Michel Canteloup, septembre 1986. Vitrine consacrée aux travaux de Michel Leiris au musée de L'Homme, exposition Leiris \& Co. Picasso, Masson, Miró, Giacometti, Lam, Bacon... Centre Pompidou-Metz (avril-septembre 2015). Cl. Jean Jamin.
2. Voir Sigmund Freud, Délire et rêves dans la "Gradiva" de Jensen, trad. de l'allemand par Marie Bonaparte, Paris, Gallimard, 1949 [1931].

\section{Gradhiva - Pouvez-vous revenir sur la création de Gradhiva?}

Jean Jamin - Gradhiva? Ça s'est fait au Totem. Le Totem était le bistrot du musée de l'Homme, au rez-de-chaussée, côté jardins du Trocadéro. Michel Leiris et moi, depuis 1977, nous nous y rencontrions tous les matins pour prendre force tasses de café et de décaféiné, y réinventions le monde autant que l'ethnologie, parlions littérature, opéra, jazz et politique ou, simplement, météo, cuisine et vins fins. C'est donc là que Leiris et moi nous avons lancé l'idée d'une revue et on s'est dit, comme ça, comme on abat une carte: «Pourquoi pas l'appeler Gradiva? » II connaissait Gradiva parce que c'était devenu une sorte d'égérie surréaliste. André Breton possédait une petite galerie à la fin des années 1930, rue de Seine $\left(\mathrm{Vl} \mathrm{l}^{\mathrm{e}}\right)$, qui s'appelait «Gradiva» et il avait fait dessiner la porte de cette galerie par Marcel Duchamp. En 1939, André Masson, un des proches amis de Leiris (et même son mentor), avait peint un tableau intitulé Gradiva, d'inspiration encore très surréaliste, alors qu'il avait quitté le mouvement avec fracas en 1929 (fig. 7). Et puis il y eut surtout Freud et son fameux essai sur la Gradiva de Wilhelm Jensen². Mais Leiris et moi on s'est dit que, nonobstant l'attirance du nom, sa symbolique («celle qui avance»), son imaginaire, son emprise, sa chronique, il fallait justifier ce titre - Gradiva - pour qu'il puisse s'appliquer à une revue d'anthropologie et non de littérature, de musique ou d'histoire de l'art. Et c'est là que nous est venue l'idée de chercher un acronyme qui, j'avoue, nous demanda pas mal d'efforts, mais sans que, avec le temps, il apparaisse tiré par les cheveux puisqu'il fonctionne encore (même si, pour ses lecteurs actuels, le sens s'est quelque peu perdu): Groupe de recherche et d'analyse documentaire sur l'histoire et les variations de l'anthropologie. Un $\mathrm{H}$ avait donc été introduit. Et le coup de génie de Jean-Michel Place, qui a été un remarquable éditeur 
et concepteur graphique, et qui fut notre premier soutien technique et commercial, c'est d'avoir fait composer le $\mathrm{H}$ en oblique, en italique. Ça faisait penser à la Gradiva de Jensen - qui avait cette démarche si particulière représentée sur et par un bas-relief et qui fascina le jeune archéologue Norbert Hanold, personnage principal du roman. Et voilà la revue partie. Le premier numéro est sorti à l'automne 1986, il comprenait une quarantaine de pages, le deuxième en mars 1987 était un peu plus copieux; le dos carré et cousu n'est apparu qu'à partir du numéro 6 (1989).

\section{G. - Vous avez écrit dans ce deuxième numéro que le $\mathbf{H}$ ren-} voyait à la fois à "histoire» et à «homme", comme si vous anticipiez votre itinéraire ${ }^{3}$ de Gradhiva à L'Homme. Et la lettre H était toujours composée en italique et en rouge dans les titres du sommaire.

J.J. - Suivant ou retraçant cet itinéraire, j'ai beaucoup appris auprès de Leiris et de Place, car, en 1985, je n'avais qu'une fort mince pratique des revues et de l'édition. J'avais fait quelques trots d'essai auprès de Gérard Lenclud qui était à la tête d'Études rurales, et participé à la préparation et la coordination de trois numéros ${ }^{4}$. J'avais également été impliqué de près dans l'édition du volume de la revue littéraire L'Ire des vents (n³-4, 1981) entièrement consacré à l'œuvre de Leiris. Quant à celui-ci, il avait une longue expérience des revues et un goût très sûr, en même temps qu'un amour du texte et un sens remarquable de la mise en page (fruit sans doute de sa longue fréquentation des ateliers de ses amis peintres, dessinateurs et sculpteurs), sans parler de son penchant, comme nombre de surréalistes, pour la photographie (en 1929, il avait même formé le projet de devenir reporter-photographe). Le principe que nous avions retenu était de ne publier que des photos en noir et blanc, qui nous semblaient marquer plus de distance entre le texte et les illustrations, et, en même temps - comme pour la Gradiva de Wilhelm Jensen - leur donner plus de corps et de relief, comme si l'un devait littéralement se fondre dans les autres, les rapports typographiques renvoyer aux rapports de contraste, et les titres en Univers gras et corps 26 à des entrelacs d'ombre et de lumière, comme dans les films expressionnistes de Murnau ou de Lang. On avait alors accès à la photothèque du musée de l'Homme, pas mal sous-exploitée, où l'on puisait ce qu'on voulait sans avoir de droits à acquitter. Leiris, à l'époque où il travaillait à la revue Documents, avait consulté toutes les agences photographiques de Paris, et il avait pour principe de ne pas utiliser les photos comme des illustrations mais d'en faire des additions au texte. Au moment de la fondation de Gradhiva, j'avais gagné, toujours avec l'aide de Leiris, une petite expérience dans le domaine de l'édition scientifique grâce à la collection «Les Hommes et leurs signes» que nous avions fondée au Sycomore à la fin des années 1970 (une maison d'édition libanaise installée en France) et qui a eu pignon sur rue de 1978 à 1984.

\section{G. - Y avait-il une répartition des rôles entre Leiris et vous?}

J.J. - Non, on concevait les numéros ensemble, toujours au Totem. À l'époque existaient ce qu'on appelait des «tables lumineuses": on recevait des bromures de la photocomposition, et on collait le texte plus les images,
3. Voir Jean Jamin, "Les chemins de la Gradiva », Gradhiva 2, $1987: 1-6$

4. Voir "La chasse et la cueillette aujourd'hui",

Études rurales 87-88, 1982 ; «Ethnographie de la violence ", Études rurales 95-96, 1984; «Le texte ethnographique ", Études rurales 97-98, 1985. 


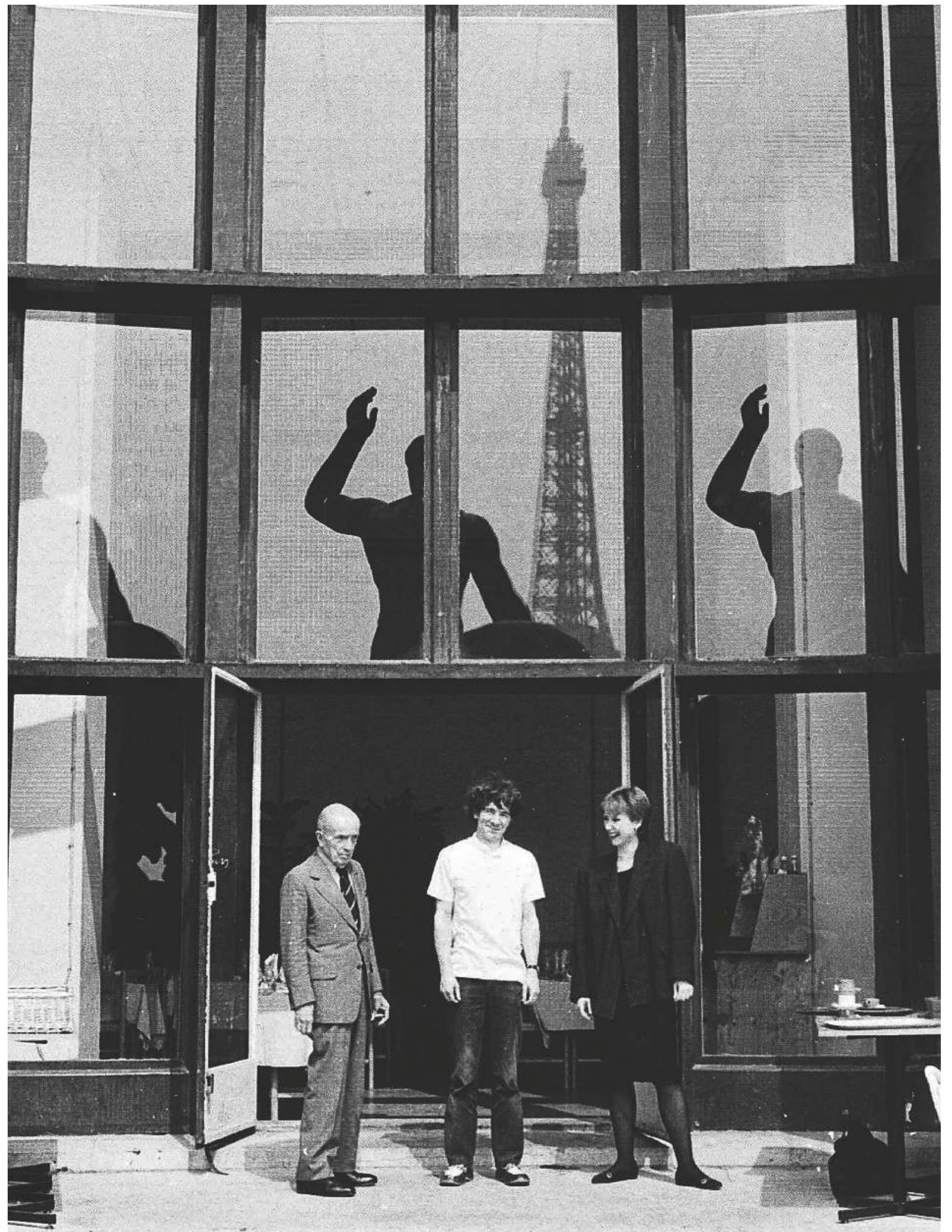


découpées au cutter, sur des canevas posés à même cette table. Ensuite je revenais chez Jean-Michel Place et on passait des nuits entières à peaufiner et caler le montage de la revue avec Michel Mousseau, le graphiste (dont je reparlerai plus tard), et à prendre les films pour l'impression. Le passage à l'informatique, au traitement de texte, à la PAO, ce fut beaucoup plus tard, après la mort de Leiris en 1990.

\section{G. - Leiris a écrit qu'il s'investissait peu dans la revue}

\section{Documents...}

J.J. - Ce n'est pas tout à fait exact, il y a beaucoup travaillé (et pas mal écrit), mais la revue Documents n'a duré que deux ans. Sans doute est-ce cela qu'il voulait dire ${ }^{5}$. II n'assistait pas au conseil de rédaction de Gradhiva, mais il demandait à lire les articles, les évaluait, proposait des corrections, s'impliquait dans la mise en page de la revue et le choix des photographies. Il était très méticuleux, tatillon parfois.

\section{G. - Comment choisissiez-vous les photographies?}

J.J. - On cherchait des photographies qui de préférence n'avaient jamais été exposées ni publiées. De ce point de vue, le musée de l'Homme possédait des réserves fantastiques. Dans les vieux tiroirs de la photothèque, pratiquement rien n'avait été utilisé. Il existait même des plaques photographiques qui n'avaient jamais été tirées, et que nous découvrions et emportions au studio avec une goinfrerie de gamins.

G. - Donc c'était une revue entièrement pensée au musée de l'Homme, mais sans être pour autant la revue du musée de l'Homme?

J.J. - Et l'on tenait beaucoup à ce qu'elle ne le soit pas. Par souci d'indépendance et, surtout, pour ne pas en faire une « revue de laboratoire», c'est-à-dire une sorte de bulletin paroissial où chanoines et ouailles se seraient arrangés d'avance et congratulés sans relâche. Non sans quelques difficultés avec la direction du Laboratoire d'ethnologie, Gradhiva a pu se faire avec l'aide d'une technicienne, Catherine Krantz. Grâce à Michel Izard et Maurice Godelier, j'avais obtenu un financement du Centre national de la recherche scientifique (CNRS) qui me permettait d'être matériellement indépendant par rapport au Laboratoire d'ethnologie. Quelques mois après la sortie du deuxième numéro, le Centre national du livre nous apporta son concours. Parallèlement à Gradhiva, avait été fondé au musée de l'Homme un département d'archives de l'ethnologie, et au CNRS, créée une recherche coopérative sur programme (RCP) intitulée «Histoire et épistémologie du savoir ethnographique » dont Jean-Pierre Digard fut le rapporteur, regroupant une quinzaine de chercheurs de plusieurs laboratoires franciliens et dont la responsabilité m'avait été confiée. En lien avec le département d'anthropologie de Berkeley (Paul Rabinow) et celui de Santa Cruz (James Clifford), une partie de cette équipe participa à deux colloques à l'université de
5. Voir Michel Leiris,

"De Bataille l'Impossible

à l'impossible Documents ", in Brisées, Paris, Gallimard, 1992 [1966] : 288-299;

Jean Jamin, «Documents revue. La part maudite de l'ethnographie ", L'Homme 151, 1999: 257-266. ci-contre

fig. 2

De gauche à droite:

Michel Leiris, Jean Jamin,

Clémentine Deliss sur la terrasse du bar-restaurant Le Totem au moment de la fondation de Gradhiva, musée de l'Homme, Paris, juillet-août 1986. DR. 
6. Chacune faisait l'objet d'un volume collectif, plutôt que catalogue, auquel nombre de nos collègues anthropologues furent conviés à écrire de courts essais sur le thème de l'exposition, et ceci en toute liberté. Tous ces ouvrages sont malheureusement épuisés.

7. Voir Patrick Ferla et Jacques Hainard, L'Ethnographie en cent images ou l'Album de Jacques Hainard, Gollion (Suisse), In Folio, 2010. Né en 1943 dans le canton de Neuchâtel, africaniste de formation, Jacques Hainard a dirigé le musée d'Ethnographie de Neuchâtel de 1980 à 2006.

8. On lui doit l'un des premiers colloques internationaux consacrés à l'histoire de l'ethnologie (CNRS, EHESS, Maison des sciences de l'Homme, Sèvres, novembre 1981) : voir Britta RuppEisenreich (dir.), Histoire de l'anthropologie: $X V I^{e}-x I x^{e}$ siècles, Paris, Klincksieck, 1984, et, avec Patrick Menget (dir.), "L'anthropologie: points d'histoire ", L'Ethnographie 90-91, 1983. Voir également Jean Jamin, «L'histoire de l'ethnologie est-elle une histoire comme les autres?", Revue de synthèse 3-4, 1988 469-483.
Californie au milieu et à la fin des années 1980 sur une thématique plus épistémologique, procédant en quelque sorte par antiphrase («Anthropologie et modernité»).

\section{G. - Quel a été le rôle de Jacques Hainard dans la création de la revue?}

J.J. - Techniquement limité, mais intellectuellement et esthétiquement important. Jacques Hainard était à l'époque directeur du musée d'Ethnographie de Neuchâtel en Suisse. II en a été le muséographe perturbant, dynamique, créatif, et il fut un concepteur et un agitateur d'idées de tout premier ordre, et aussi et surtout un grand ami. Il cherchait à démontrer, contre tout essentialisme et ontologisme, que l'objet ethnographique variait en fonction des regards que, dans l'histoire, selon les circonstances, les périodes, les modes, on portait sur lui, non sans que ceux qui le mettaient en réserve ou en vitrine ne soient eux-mêmes soumis à ces effets de texte et de contexte. Critique, l'esprit devait l'être aussi bien vis-à-vis de soi. De 1982 à 2002, je suis allé presque chaque année à Neuchâtel, où j'étais invité à participer, comme conseiller scientifique, à des expositions ${ }^{6}$ que Jacques Hainard inlassablement programmait dès le mois de novembre, plus inventives les unes que les autres, chacune toujours aussi dérangeante, de "Collections passion » au «Trou » en passant par «Le corps enjeu», «Le mal et la douleur» ou «Temps perdu, temps retrouvé»... Des expositions toutes thématiques qui transgressaient les frontières des aires dites culturelles, et, par le détour, historique ou géographique, venaient interroger notre modernité. «Objets prétextes, objets manipulés», disait Jacques Hainard, les objets ethnographiques se dépouillaient petit à petit de leur qualité d'objets-témoins dans laquelle on les avait confinés, et s'invitaient sur la table garnie des visiteurs («Le musée cannibale», suivant le titre de sa dernière exposition à Neuchâtel en 2002). Sans son soutien, son avis, ses propositions, sa perception, Gradhiva n'aurait sans doute pas eu la même allure ni la même histoire (il m'avait dit qu'en cas de besoin il pourrait même financer la revue). Au fond, je m'aperçois maintenant qu'avec Gradhiva nous naviguions dans une sorte de quadrature du cercle, c'est-à-dire la transposition en deux dimensions des rapports iconiques, des rapprochements artefactuels et des associations textuelles que Jacques Hainard réalisait en trois dimensions dans ses expositions, n'hésitant pas à provoquer ni à ébranler. Donner à voir, faire entendre, et ne jamais laisser l'œil et l'esprit en repos, tel aurait pu être le credo de Jacques Hainard, tel aussi aura été, à sa suite, celui de Gradhiva 7 .

\section{G. - Comment expliquez-vous rétrospectivement le succès de cette revue?}

J.J. - À l'époque, l'histoire de l'anthropologie, ou plutôt de l'ethnologie, n'était pas réellement un champ de recherche et d'analyse autonome, en tout cas pas en France, malgré les livres pionniers de Paul Mercier et les enseignements et travaux de Britta Rupp-Eisenreich à l'EHESS ${ }^{8}$. C'était soit des propos généraux présentés en introduction de cours ou de séminaires, 


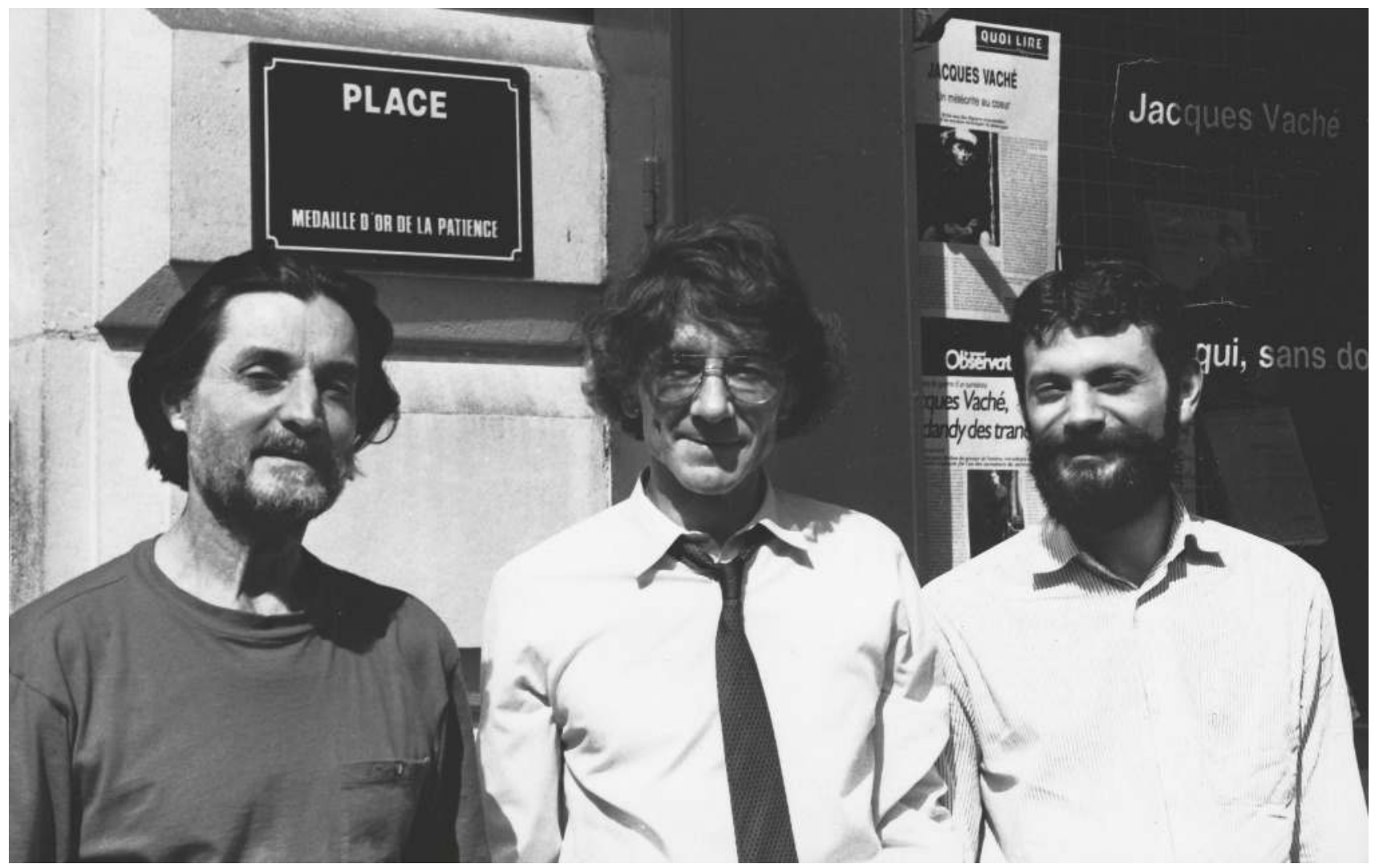

fig. 3

De gauche à droite : Michel

Mousseau (artiste-peintre, graphiste et maquettiste), Jean Jamin, Vincent Gimeno (secrétaire d'édition) devant les Éditions Jean-Michel Place, 10 rue Pierre-etMarie-Curie (Paris Ve) au moment de la préparation du numéro 5 de Gradhiva en juillet 1988, Paris, DR. 
9. Voir Jean Jamin "The Making of Ethnographic Texts. Santa Fe, 16-20 avril 1984 ", Gradhiva 1, 1986: 31

10. Voir Jean Jamin et Françoise Zonabend, (dir.), "Le texte ethnographique ", Études rurales, 97-98, 1985.

11. Voir Giordana Charuty, Michèle Coquet et Jean Jamin, «In Memoriam: Monsieur Fabre n'est jamais là ", Gradhiva 23, 2016: 3-25

12. Voir James Clifford "On Ethnographic Surrealism ", Comparative Studies in Society and History 23, 1981, repris dans Malaise dans la culture: l'ethnographie, la littérature et l'art au $x x^{e}$ siècle, trad. de l'anglais par Marie-Anne Sichère, Paris, ENSBA, 1996 [1988]: 121-163; et Jean Jamin "L'ethnographie, mode d'inemploi: de quelques rapports de l'ethnologie avec le malaise dans la civilisation ", in Jacques Hainard et Roland Kaehr (dir.), Le Mal et la douleur Neuchâtel, Musée d'Ethnographie, 1986 : 45-79.

13. Voir Jeanne FavretSaada, "Être affecté", Gradhiva 8, 1987 : 3-9; Françoise Zonabend, «De l'objet et de sa restitution en anthropologie ", Gradhiva 16, 1994: 3-14.

14. Voir Robert Musil, L'Homme sans qualités, trad. de l'allemand par Philippe Jaccottet, Paris, Seuil, 2004 [1930], 2 vol. soit des considérations de fin de carrière, mais ça n'était pas conçu comme un domaine aussi fécond et heuristique que pouvait l'être l'histoire de la philosophie ou de la sociologie. C'était peut-être dû au fait que l'ethnologie était une discipline encore jeune, bien qu'aux États-Unis l'histoire de l'anthropologie se fût vite imposée comme un champ de recherche à part entière, fouillé et développé entre autres par George W. Stocking à l'université de Chicago. De leur côté, des chercheurs comme Clifford Geertz, James Clifford, Paul Rabinow, Herman Lebovics, pour ne citer qu'eux, avaient montré la nécessité de faire de l'histoire de l'anthropologie à partir du moment où se mettait en place une approche réflexive de la discipline, et qu'on prenait à bras le corps ce qu'on peut appeler la «fabrique du texte ethnographique».

\section{G. - Ce débat lancé par le colloque intitulé "Writing Culture" sur le postmodernisme, vous lui avez fait une place dans Gradhiva?}

J.J. - Oui, le premier compte rendu en français du livre qui en est issu est paru, je crois, dans Gradhiva. Ce colloque avait eu lieu à Santa Fe en 1984, et j'en avais fait une courte recension ${ }^{9}$. II y a eu en même temps le numéro d'Études rurales qui s'intitulait «Le texte ethnographique » que j'ai dirigé avec Françoise Zonabend ${ }^{\mathbf{1 0}}$, après le colloque de Toulouse sur les «Voies nouvelles en ethnologie de la France» que Daniel Fabre avait organisé ${ }^{11}$. Ce tournant réflexif a connu des excès, comme le «surréalisme ethnographique» dont a parlé James Clifford et que j'ai vivement (sans doute trop vivement) critiqué ${ }^{\mathbf{1 2}}$, mais il a aussi secoué les consciences et apporté des perspectives non négligeables, en particulier sur la construction de l'objet, sur les méthodes de l'enquête ethnographique, les processus d'interlocution et de restitution ${ }^{\mathbf{1 3}}$.

\section{G. - Le sous-titre de Gradhiva - revue d'histoire et d'ar-} chives de l'anthropologie - allait bien dans ce sens.

J.J. - Sur ce plan-là Gradhiva a bien tenu la route. Je crois que c'était, à l'époque, la seule revue d'anthropologie entièrement vouée à l'histoire, à l'historiographie et à l'épistémologie de la discipline, et qui consacrait une partie non négligeable de ses colonnes à la publication d'archives et de matériaux inédits, tirés de fonds privés ou publics. Le département d'archives de l'ethnologie sur lequel elle s'adossait de même que la RCP «Histoire et épistémologie du savoir ethnographique " permettaient à la revue de couvrir tout un champ de recherche qui n'était plus de l'ordre de la garniture ou de l'anecdote, mais cherchait à se porter au cœur de la vie savante en en restituant les enjeux cruciaux ou simplement les à-côtés ou les «entre-deux", selon les mots de Robert Musil ${ }^{\mathbf{1 4}}$, qui ne sont pas moins cruciaux. Peut-être est-ce là qu'on retrouvait le «tournant réflexif », dans la mesure où cette vie savante était aussi traversée de courants sensibles, artistiques, littéraires, musicaux, que l'ethnologie, en tant que science de l'homme, ne pouvait négliger. Principalement axée sur l'histoire de l'ethnologie française, sans pour autant s'interdire des incursions dans d'autres écoles nationales, Gradhiva restituait ce sur quoi Claude Lévi-Strauss avait insisté, à savoir cette physionomie originale qu'avait donc l'ethnologie française, à l'écoute de résonances 
entre les arts plastiques et la musique, le savoir et la poésie, le culte des faits et l'imagination poétique ${ }^{\mathbf{1 5}}$. C'est cela que m'avait apporté Leiris et qu'il apportait dans Gradhiva: à savoir que «l'exotique» n'a pas de fin, contrairement à ce que pensent certains; il peut être - il est - à l'origine du poétique, ne serait-ce que parce qu'il révèle ce qu'il y a d'autre en soi, comme nous le rappelle la célèbre formule de Rimbaud: «Je est un autre». Ce qui m'amena d'ailleurs à m'intéresser en anthropologue à l'œuvre de Victor Segalen ${ }^{16}$, et à me replonger, grâce à Leiris, dans celles de Jules de Gaultier et de Marcel Schwob ${ }^{\mathbf{1 7}}$. En ce sens, il ne pouvait pas y avoir d'ethnographie sans poésie; les images dans Gradhiva, leur mise en place et en page, devaient en être la manifestation et la preuve (comme cela avait été le cas avec la revue Documents).

\section{G. - Comment êtes-vous passé d'un H à l'autre?}

J.J. - Disons de l'oblicité gradhivienne à la raideur toute structuraliste de L'Homme! Eh bien, ce fut en 1996. Jean Pouillon, qui était depuis trente-cinq ans à la tête de la revue L'Homme éditée au Laboratoire d'anthropologie sociale du Collège de France, venait d'avoir 79 ans, et voulait quitter ses fonctions. II y eut un comité d'une dizaine de personnes qui s'était mis en place sous la direction de Jacques Revel, à l'époque président de l'EHESS dont L'Homme dépendait administrativement et financièrement, pour lui trouver un successeur. Aucun accord ne se fit jour sur les quelque cinq ou six candidats qui avaient été sollicités ou s'étaient spontanément présentés. Jean Pouillon refusait, par ailleurs, le principe d'un comité de direction ou d'un «comité scientifique " prenant les décisions et définissant la politique éditoriale de la revue. II se reportait à l'histoire même de celle-ci où Claude Lévi-Strauss, Émile Benveniste et Pierre Gourou, les fondateurs, se sont d'abord présentés comme un «comité de rédaction», dont Jean Pouillon n'était pas le rédacteur en chef (il détestait ce terme de «chef»), mais le secrétaire général, à l'image de celui qui est en fonction à la tête d'un syndicat, d'un parti politique... ou de l'ONU...

\section{G. - Mais il n'avait jamais fait de terrain et n'avait jamais émis le regret de ne pas en faire.}

J.J. - Bien que diplômé de philosophie, il n'était pas universitaire. II venait d'ailleurs. II a fait un peu de terrain en Éthiopie et au Tchad, avec le soutien du Laboratoire d'anthropologie sociale. De 1961, année de sa création, à 1996, L'Homme s'est imposé grâce à lui dans le paysage anthropologique français, et même international. Donc en 1996, il y avait eu beaucoup de désaccords dans ce comité destiné à lui trouver un successeur. Un soir Marc Augé m'a dit: “Ce serait bien que ce soit toi, tu mettrais au moins tout le monde d'accord. " Je n'étais pas chaud, même à cette heure avancée de la nuit où après deux whiskies (peut-être trois) nous devisions - déjà - sur l'avenir des revues d'anthropologie. Je «possédais " Gradhiva où je me sentais de fait plus libre, non pas dans le choix des articles auxquels s'appliquaient les mêmes critères qu'à L'Homme, mais dans les jeux de maquette et de mise en page. Ceux de L'Homme me semblaient d'une austérité tout académique, pour tout dire plate et ennuyeuse. Mais l'idée
15. Voir Claude LéviStrauss, «Allocution à l'occasion de l'hommage à Georges Henri Rivière rendu par le musée national des Arts et Traditions populaires" (26 novembre 1986), Ethnologie française 2, 1986: 127-133.

16. Jean Jamin fut encouragé à lire Segalen par André Schaeffner et sa femme Denise Paulme qui avait été sa directrice de recherche à l'Office de la recherche scientifique et technique outre-mer (Orstom, actuel IRD), et à qui il avait dédié sa monographie sur la tenderie aux grives (voir Jean Jamin, La Tenderie aux grives chez les Ardennais du plateau, Paris, Institut d'ethnologie, 197).

17. Voir Jean Jamin, "Fictions haut régime. Du théâtre vécu au mythe romanesque", L'Homme 175-176, 2005 : 165-202. 


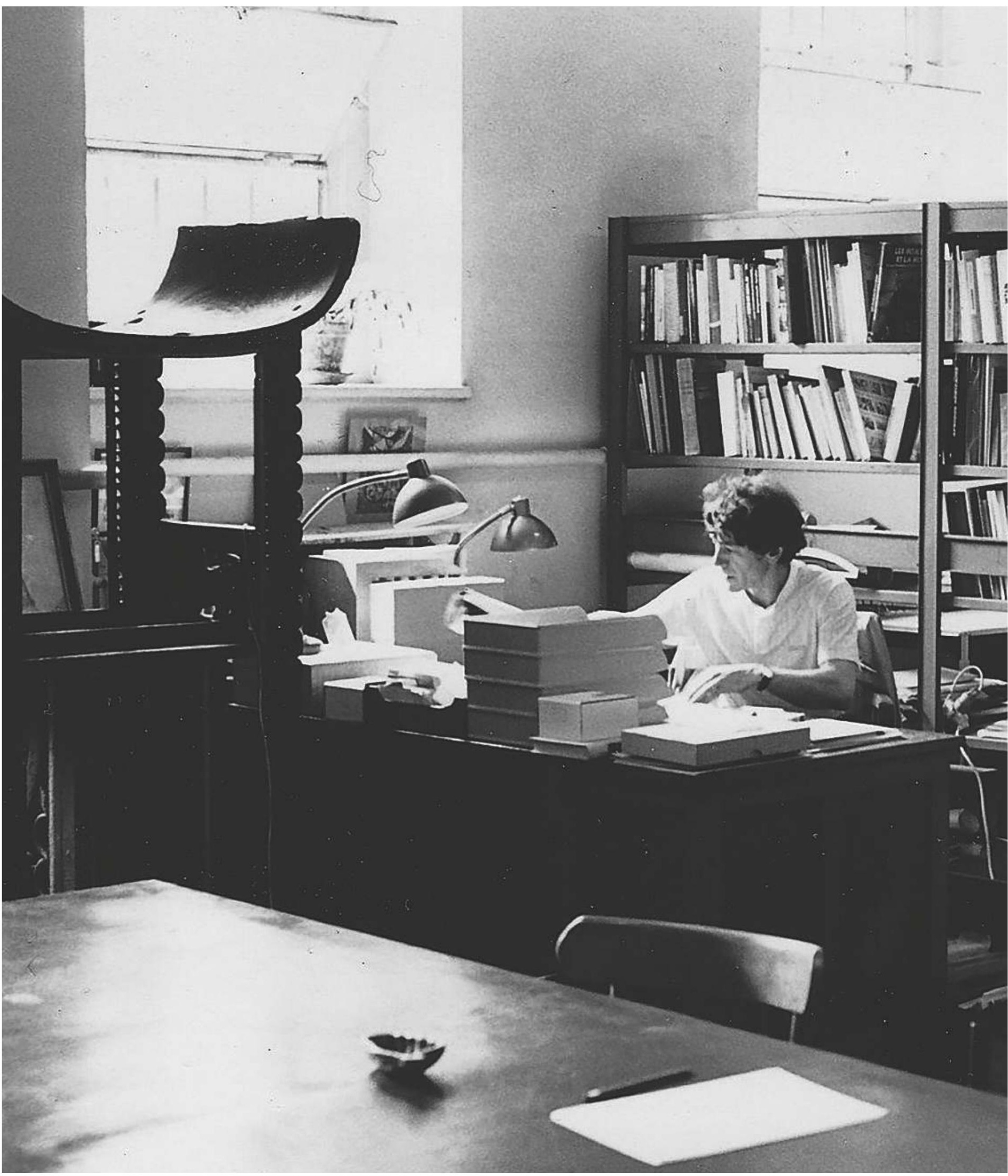




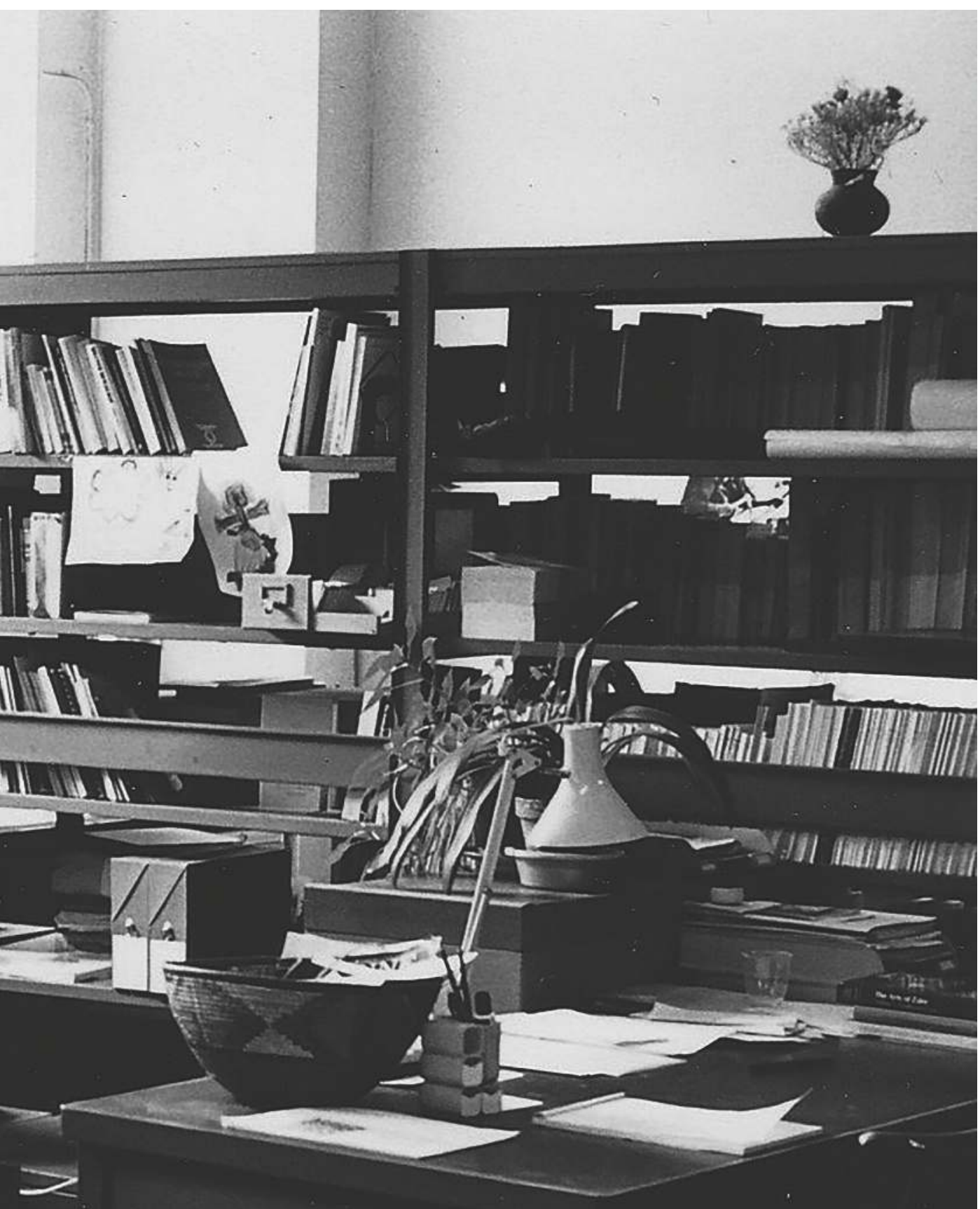

ci-contre et

pages 206-207

fig. 4

Secteur du Département d'Afrique noire dans les sous-sols du musée de L'Homme où seront momentanément hébergés (de 1985 à 1988) le

Département d'Archives de l'ethnologie, la RCP n 847 du CNRS et la rédaction de Gradhiva, Paris, juillet 1986. DR. 
18. Voir Jean Jamin, La Tenderie aux grives [...], op. cit., 1979 ; id., Les Lois du silence: essai sur la fonction sociale du secret, Paris, Maspero, 1977

19. Voir Jean Jamin et Françoise Zonabend (dir.), Gradhiva 30-31, 2001-2002. qu'on en pût changer - ce que me confirmèrent Marc Augé, Françoise Héritier, Élisabeth Coppet-Rougier, Jacques Revel et Jean Pouillon qui devinrent très vite des interlocuteurs - finit par me convaincre que le défi méritait d'être relevé. Ce que, après avoir accepté, je fis en m'adressant au graphiste de Jean-Michel Place, Michel Mousseau, qui recomposa complètement la maquette de L'Homme, tant au niveau de la mise en page que de la typographie ou du choix des polices de caractères, et en faisant passer le secrétariat de rédaction de la revue de l'ère du Tipp-Ex à celle de I'XPress, et en remplaçant les machines à boule d'IBM par les écrans encore nains des Macintosh Plus. De ce point de vue, L'Homme fut la première revue de l'EHESS à être entièrement conçue, techniquement et bien sûr intellectuellement, en «interne», dans les bureaux et sur les ordinateurs de la rédaction. C'est-à-dire que l'imprimeur ne recevait que des fichiers «bons à clicher». Outre le gain financier, cela permettait d'avoir une évaluation immédiate de la mise en page des textes et de leur éventuelle évolution typographique : en somme un work in progress (ce que devrait être toute revue). Entre-temps, je demandai à Françoise Zonabend, qui, depuis 1986, avait suivi de près les travaux de la RCP n 847 du CNRS sur l'histoire de l'ethnologie et pas mal de dossiers publiés dans Gradhiva, de me succéder à la tête de cette revue. Elle a accepté, et elle l'a dirigée pendant dix ans, jusqu'à sa reprise par le musée du quai Branly. Je restais directeur de la publication, mais ce n'était là qu'une fonction juridique.

\section{G. - Quand vous avez pris la direction de L'Homme, quelles étaient vos relations avec Claude Lévi-Strauss?}

J.J. - Je le connaissais un peu, mais je me situais plus dans la mouvance et le courant d'idées initiés par Georges Balandier que dans ceux du structuralisme. J'avais peu de relations intellectuelles ou amicales avec de proches collaborateurs ou étudiants de Claude Lévi-Strauss, si ce n'était avec Françoise Héritier, Françoise Zonabend ou Michel Izard. Mais LéviStrauss a toujours été très attentif; il avait lu mon travail ethnographique sur la tenderie aux grives et mon essai sur Les Lois du silence ${ }^{18}$... Quand il a appris la fondation de Gradhiva, il m'a fait part de son intérêt. De même lorsque s'est constitué, au musée de l'Homme, le département d'archives de l'ethnologie, il s'en est ouvertement réjoui. Quand j'ai repris L'Homme, j'étais allé lui présenter la nouvelle maquette. Je n'en menais pas large, devinant ses goûts plutôt conservateurs, mais un «C'est très bien », et «Le petit bonhomme nous fait coucou! ", faisant allusion à la vignette de L'Homme que le maquettiste Michel Mousseau avait habilement disposée de part et d'autre de la tranche du volume, avaient suffi à me, à nous rassurer. Dans les années qui ont suivi, je n'ai jamais eu la moindre critique de sa part quant au contenu de la revue: il la lisait attentivement, au besoin nous donnait un article ou un compte rendu d'ouvrage.

G. - Le thème des archives n'apparaît dans le titre d'un numéro spécial ${ }^{19}$ de Gradhiva qu'en 2001 alors que c'était un sujet central pour la revue dès sa fondation. Est-ce que le travail pour L'Homme vous a permis de mieux définir la spécificité de Gradhiva? 
220

entretiens

Georges BALANDIER

422 owork 1286 ,

Chus jim jamin,

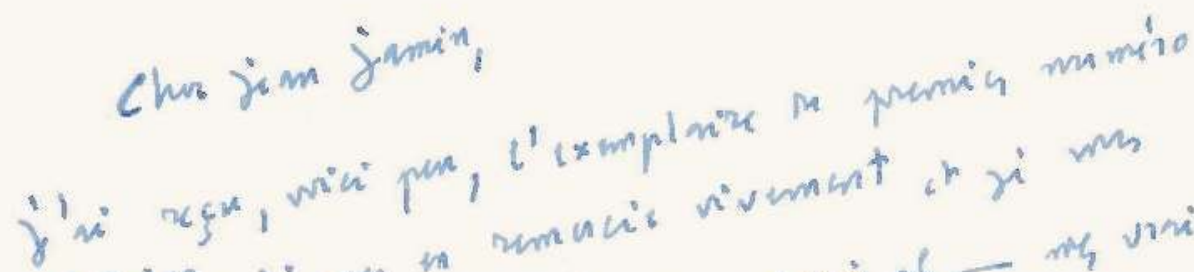

$$
\text { fikicitrions. }
$$

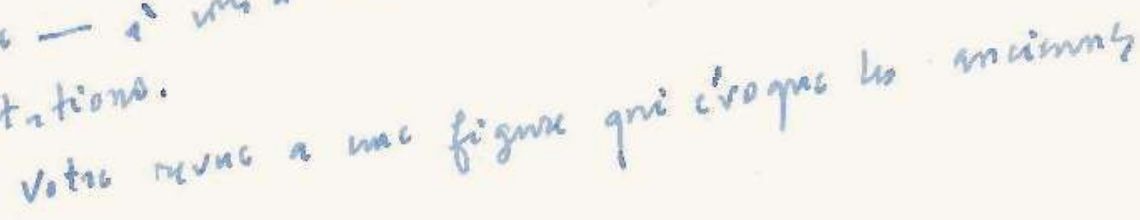

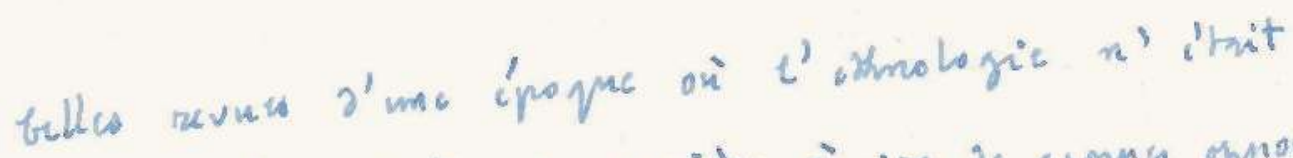

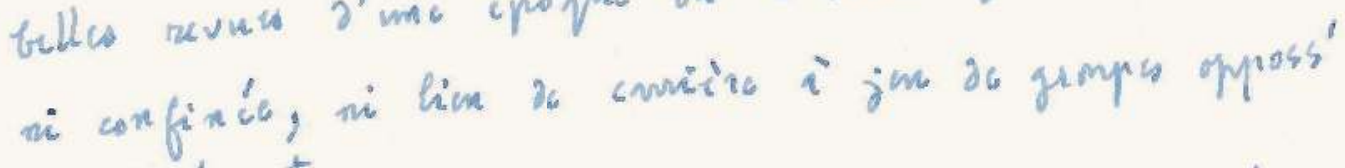
it intolis mits.

$$
\text { cintolinant. }
$$

$\partial_{c} t^{\prime}$ spint ar a la smesitilit'. vots propre textc, insolits

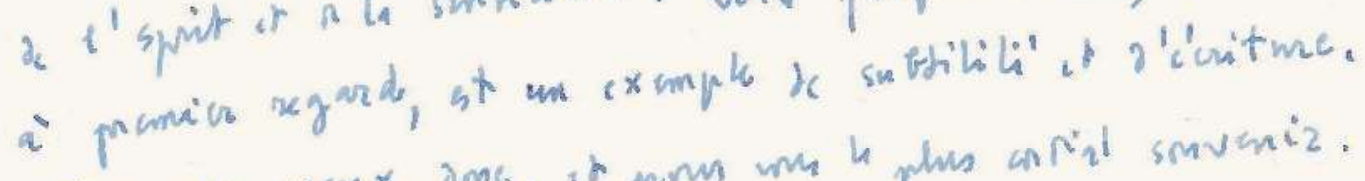

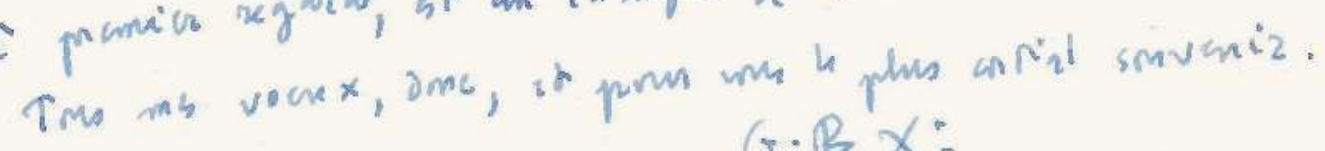

$$
G \cdot B X:-
$$

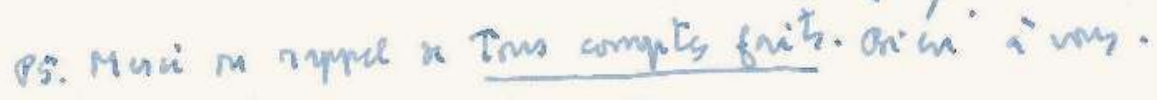


J.J. - Sans doute la réflexion proprement dite n'était-elle pas suffisamment mûre, nous étions surtout soucieux de publier d'abord des matériaux. D'ailleurs, le titre de la présentation que Françoise Zonabend et moi avions proposé est-il une sorte de clin d'œil, voire d'ironie ${ }^{20}$. Et j'avais été très influencé par ce qu'a réalisé George Stocking au États-Unis. Au tout début des années 1980, dans des circonstances rocambolesques (grâce, si j'ose dire, à une fuite des toilettes du rez-de-chaussée du musée de l'Homme dont les eaux bien peu claires s'étaient déversées dans les sous-sols où nous avions nos bureaux, remises et réserves), j'ai redécouvert les archives du musée d'Ethnographie du Trocadéro, qui ont du reste permis à notre collègue Nélia Dias, alors jeune étudiante de Pierre Nora et de Denise Paulme, de terminer sa thèse sur la fondation de ce musée ${ }^{\mathbf{2 1}}$. C'est comme cela que je suis tombé sur les dossiers de la mission Dakar-Djibouti, dont j'ai fait, par la suite, mon fer de lance $\mathbf{2 2}$. Gradhiva permettait de publier ces dossiers par périodes, morceaux et tranches, ou par catégories; les premières lettres de Denise Paulme et Deborah Lifchitz, envoyées de Sanga (Mali), chez les Dogon en 1935, ont paru dans Gradhiva ${ }^{23}$. II commençait à y avoir un intérêt des étudiants pour l'histoire de l'anthropologie; je citais Nélia Dias, mais il faudrait aussi mentionner Thomas Beaufils qui avait fait sa thèse sur l'anthropologie néerlandaise et sur les rapports entre Josselin de Jong et LéviStrauss; puis, plus tard, Éric Jolly et Marianne Lemaire ${ }^{\mathbf{2 4}}$, Benoît de L'Estoile ${ }^{\mathbf{2 5}}$, Emmanuelle Sibeud ${ }^{26}$.

\section{G. - Aux États-Unis, y avait-il une revue analogue?}

J.J. - Stocking avait une sorte de bulletin ronéoté mais pas de revue. II avait cependant créé une magnifique collection qui, au fond, jouait le rôle d'une revue apériodique sur l'histoire de l'anthropologie $\mathbf{2 7}$.

\section{G. - Quand vous avez pris la succession de L'Homme, avez-} vous essayé d'y ajouter la ligne éditoriale de Gradhiva?

J.J. - Oui, il y eut même une petite concurrence entre Françoise Zonabend et moi parce que je commençais à publier des images dans L'Homme. Je n'ai pas non plus hésité à publier des textes d'archives (correspondances, manuscrits, fiches et notes de terrain, etc.). Non point avec une quelconque envie de rivaliser mais par un souci de cohérence, considérant qu'une revue généraliste telle que L'Homme ne pouvait se dispenser d'apporter des matériaux à l'histoire de la discipline qu'elle représentait. Le passage de Gradhiva au musée du quai Branly et son recentrage sur l'anthropologie et l'histoire de l'art évitèrent en somme de trop se marcher sur les pieds.

\section{G. - Une autre inflexion, c'est l'introduction de la littérature} et de la musique.

J.J. - II y avait eu un numéro dirigé par Daniel Fabre et Jean Pouillon sur anthropologie et littérature ${ }^{\mathbf{2 8}}$, qui était mal passé dans la communauté professionnelle. Cependant, quand, avec François Flahault et Nathalie Heinich,
20. Voir Jean Jamin et Françoise Zonabend, "Archivari", Gradhiva 3031, 2001-2002: 57-65.

21. Voir Nélia Dias, Le Musée d'Ethnographie du Trocadéro (1878-1908) anthropologie et muséographie en France. Paris, CNRS Éditions, 1991.

22. Voir Jean Jamin, "Le cercueil de Queequeg", art. cit.

23. Voir Marianne Lemaire (éd.), Lettres de Sanga. Paris, CNRS Éditions, 2015

24. Voir leur édition savante et critique de toutes les publications scientifiques de la fameuse mission Dakar-Djibouti, 1931-1933 (Cahier DakarDjibouti. Meurcourt, bourgade de la HauteSaône, siège des éditions Les Cahiers, 2015, 1440 pages).

25. Voir Benoît de L'Estoile, Le Goût des autres : de l'Exposition coloniale aux arts premiers, Paris, Flammarion, 2007.

\section{ci-contre}

fig. 6

Lettre de Georges Balandier reçue en octobre 1986 après la parution du premier numéro de Gradhiva. Coll. Part. Cf. aussi infra, p. 227, note 36
26. Voir Jean-Loup Amselle et Emmanuelle Sibeud (dir.), Maurice Delafosse: entre orientalisme et ethnographie, l'itinéraire d'un africaniste (1870-1926). Paris, Maisonneuve et Larose, 1998.

27. "History of Anthropology ", Madison, University of Wisconsin Press, en moyenne un volume par an.

28. "Littérature et anthropologie ", L'Homme 111-112, 1989 
29. Voir Jean-Pierre Digard, "L'Homme avec et sans Pouillon ", L'Homme 143 (numéro spécial : «Histoire d'homme: Jean Pouillon "), 1997

30. Ethnologue africaniste, directeur d'études à l'EHESS et directeur du CAMC, Jean Bazin (1941-2001) est notamment l'auteur de Des clous dans la Joconde: l'anthropologie autrement, Toulouse, Anacharsis, 2008 (ouvrage posthume réunissant une grande partie de ses articles). j'ai lancé le numéro « Vérités de la fiction », ou plus tard, avec Daniel Fabre et Marcello Massenzio, préparé celui consacré à «l'auto-biographie et l'ethnobiographie ", l'approche était déjà entrée dans le champ intellectuel de l'anthropologie, je n'ose dire dans les mœurs. Nous y avons œuvré soit dans des séminaires soit dans des articles, soit encore dans des volumes collectifs. Les sociologues avaient été en avance sur nous; ils ont fait de la sociologie de la littérature bien avant que les anthropologues n'y viennent. Ils nous ont aussi devancés sur le contemporain, la ville, les prisons... Le dernier numéro qui me tient particulièrement à cœur et qui est le dernier que j'aurai dirigé avec Daniel Fabre, mort moins de trois mois après sa parution, porte sur la chanson populaire («Connaît-on la chanson?»): un domaine qui était resté dans l'antichambre des recherches ethnomusicologiques classiques et auquel Fabre et moi voulions apporter quelques lettres de noblesse, ne serait-ce qu'en nous abritant derrière le célèbre vaudeville de Beaumarchais: «Tout finit par des chansons».

\section{G. - Dans I'hommage à Pouillon, en 1997, Jean-Pierre Digard} dit que L'Homme avait la réputation d'être une revue fermée et élitiste ${ }^{29}$.

J.J. - Jean Bazin ${ }^{\mathbf{3 0}}$, qui n'était guère porté vers le structuralisme, disait que c'était une «Bastille à prendre». Elle apparaissait en effet comme la revue du Laboratoire d'anthropologie sociale, une revue structuraliste, dont les proches de Georges Balandier et les marxistes ou marxologues se sentaient ou se disaient exclus, bien plus qu'ils ne l'étaient en réalité, ne voulant ou n'osant pas soumettre des articles à Pouillon qui, pourtant, n'avait rien d'un censeur ni d'un idéologue, ni même d'un thuriféraire de la structure. L'Homme n'était et n'aura jamais été une revue «doctrinale», comme eût dit Merleau-Ponty à propos des Temps Modernes. C'était aussi une revue centrée sur l'Amérique, l'Asie et l'Océanie, mais assez peu sur l'Afrique.

\section{G. - Le comité de rédaction a-t-il beaucoup changé?}

J.J. - J'ai cherché à le renouveler par tiers tous les deux ans. Mais les contraintes administratives de parité ou de répartition Paris/Province ont compliqué terriblement ces renouvellements, du fait que le milieu professionnel des ethnologues en France est démographiquement un tout petit milieu et que le «turnover» ne peut qu'être limité. Ce faisant, il est totalement illusoire de vouloir aligner le fonctionnement de cette revue sur celui de revues américaines, telles que American Anthropologist, qui sont souvent des revues associatives comptant des centaines sinon des milliers d'adhérents. L'échelle n'est pas la même et, une fois de plus, ce sont là des erreurs technocratiques que de vouloir s'y plier. Pouillon avait parfaitement senti cela et s'en était ouvert à moi lorsque j'ai pris sa succession.

\section{G. - Y a-t-il des courants de l'anthropologie que vous repré- sentiez plus que d'autres?}


J.J. - Non, parce que si Gradhiva était ma revue, L'Homme demeurait une revue académique, alors il convenait de se mettre dans la position d'un secrétaire général qui devait non seulement s'intéresser à tous les courants de la discipline, mais aussi à ses aires culturelles et je dirais même à ses aires institutionnelles qui, au fil du temps, et comme il en est de toute segmentation, ont développé des particularités non dépourvues de susceptibilités. Il convenait de les ménager, sans pour autant renoncer à des principes qui, pour moi, ont toujours été la qualité des travaux publiés, au risque parfois de déplaire à quelques «petits maîtres » (il y en a) qui cherchaient avant tout à placer leurs étudiants et doctorants. Quoi qu'il en soit, avec L'Homme j'ai ajouté, mais je n'ai jamais rien retranché. Même le «tournant animaliste» ou «ontologiste», sur lesquels j'ai exprimé de sérieuses réserves, y ont été représentés! Ce qui n'excluait pas des réponses critiques et des débats.

G. - On pourrait discuter ce terme “ontologisme" car poser des questions ontologiques en anthropologie n'implique pas de prendre position en faveur de "l'être". Mais pouvez-vous rappeler comment Gradhiva est arrivée au musée du quai Branly?

J.J. - Hélène Fulgence avait eu un coup de foudre pour cette revue. Elle connaissait Jean-Michel Place qui, se trouvant face à de grosses difficultés de trésorerie, lui avait proposé de racheter la revue et de l'accueillir au musée du quai Branly. Je lui ai rappelé que, avec Michel Leiris, j'étais propriétaire du titre, et qu'il n'était pas question d'en faire un objet de négociation mercantile. Je suis donc moi-même allé voir Hélène Fulgence pour lui proposer le titre sans aucune contrepartie. Ça a causé un petit conflit pendant un an et demi entre Place et le musée du quai Branly, et moi, par ricochets, mais, pour le bien de la revue et sa continuité, les choses se sont apaisées, sans permettre toutefois que les numéros de la première série de Gradhiva soient mis en ligne (c'est fort dommage), Jean-Michel Place invoquant - ce qui est en partie vrai - que, si le titre ne lui appartenait pas, la mise en page quant à elle était sa propriété intellectuelle.

G. - Vous avez écrit un livre intitulé Les Lois du silence sur le rôle du secret dans la circulation de la parole et du savoir chez les chasseurs/piégeurs ardennais ou les Sénoufo. Avez-vous observé un phénomène similaire dans le fonctionnement des revues?

J.J. - Je ne crois pas que nos milieux professionnels diffèrent fondamentalement, dans leur fonctionnement quotidien et dans leurs rapports aux savoirs, des groupes et sociétés que vous évoquez. On aimerait le croire, mais, tout en étant moins exotique, cela reste une illusion. Une des «lois du silence » que je respecterai ici est, par exemple, la manière dont on m'a récemment écarté de la direction de L'Homme, pour des raisons mystérieuses et sans doute peu dicibles, et qui vérifient une des vieilles hypothèses de mon livre: à savoir que tout non-dit - non-dire - entre dans une mécanique de pouvoir et d'assujettissement dont on est le complice (en en partageant le secret) ou dont on devient la victime (quand on en est exclu). Cela semble arithmétique, mais c'est malheureusement sociologique. 
31. Voir Leiris \& Co: Picasso, Masson, Miró, Giacometti, Lam, Bacon... exposition du Centre Pompidou-Metz, Metz, 3 avril-14 septembre 2015; voir aussi le catalogue du même titre, sous la direction d'Agnès de La Beaumelle, Marie-Laure Bernadac et Denis Hollier Paris/Metz, Gallimard/ Centre Pompidou-Metz, 2015, voir enfin la note de lecture d'Éléonore Devevy, "Leiris, singulier pluriel ", Gradhiva 23, 2016: 215-226.

32. Voir Jean Jamin, Faulkner: le nom, le sol et le sang, Paris, CNRS Éditions, 2011: 60 sq.

33. Voir Comité information Sahel, Qui se nourrit de la famine en Afrique? : le dossier politique de la faim au Sahel. Paris, Maspero, 1973; voir également Gérard Althabe, Les Fleurs du Congo. Paris, Maspero, 1972: Jean-Louis Boutillier, "C'était une sorte de bulle... », L'Homme 185186, 2008: 33-38.

34. Allusion à l'ouvrage de Pierre Jalée, Le Pillage du tiers-monde: étude économique, Paris, Maspero, 1965.

35. Voir notamment Françoise Héritier, Sida: un défi anthropologique, Paris, Les Belles Lettres, 2013.

\section{G. - Donc vous n'avez pas regretté de ne plus faire du terrain, car votre terrain c'était les anthropologues?}

J.J. - D'abord je ne voulais ou ne pouvais plus faire de terrain (en partie à cause de problèmes de santé), c'est la raison pour laquelle j'étais content d'avoir ouvert ce chantier sur l'histoire de l'anthropologie. Surtout, j'avais découvert cet autre terrain qu'étaient les archives (et non pas les anthropologues - l'ethno-biographie est une autre affaire). Ensuite, j'ai rencontré Michel Leiris, et je suis rentré dans l'intimité de cet homme, non seulement de sa personne et personnalité que j'ai vue pratiquement chaque jour pendant près de quinze ans, et à qui me liait une très profonde amitié, mais de son œuvre littéraire et ethnologique dont je suis l'exécuteur testamentaire et dont j'ai réalisé ou supervisé des travaux d'édition savante et critique. La grande exposition sur Michel Leiris qui a eu lieu l'an dernier au Centre Pompidou-Metz, avec d'ailleurs la participation du musée du quai Branly, et que vous avez pu visiter, m'a permis de renouer à la fois avec le musée de l'Homme et le musée d'Ethnographie de Neuchâtel, de retrouver en somme cette effervescence de ce qui s'expose ou de ce qui se dit en trois dimensions ${ }^{\mathbf{3 1}}$.

G. - Vous avez employé la notion de "désillusion biographique" à propos de William Faulkner, en disant que I'homme n'était pas à la hauteur de l'œuvre ${ }^{32}$. Est-ce que cela s'applique au secrétaire général d'une revue?

J.J. - Mais de quelle œuvre s'agit-il? Une revue est un organe collectif, un ensemble, une addition, une organisation d'articles qui tous font partie d'une autre œuvre, qui reste personnelle. Le secrétaire général n'est qu'un metteur en page (comme il y a des metteurs en scène) ou - la métaphore me semble plus juste - un chef d'orchestre, avec ses pupitres, ses solistes, ses cadences, ses silences. S'il existe une «désillusion biographique», elle a trait non pas aux non-dits (ou aux zones d'ombre) de la vie mais aux ratés de l'orchestration et de l'interprétation. II y a des choses, oui, que je regrette de ne pas avoir faites. II y a des prises de position politiques qui auraient dû ou pu être publiées dans des éditoriaux de numéros de L'Homme. Mais c'est une rhétorique difficile à maîtriser que d'intervenir dans le débat public. Je n'ai pas fait non plus de numéro sur des sujets de société, et je le regrette également. Somme toute, j'estime avoir été plutôt timoré en comparaison de la génération des Balandier, Terray, Augé, Boutillier, Althabe, Héritier, Meillassoux, etc., qui, vis-à-vis du colonialisme, du néocolonialisme ${ }^{33}$, du «pillage du tiers-monde ${ }^{\mathbf{3 4}}$, ou des pandémies comme le sida et des stigmatisations auxquelles elles ont donné lieu ${ }^{35}$, ont eu des positions tranchées, et ont cherché à réserver aux ethnologues une place dans les affaires de la cité. Les temps ont certes changé, les priorités politiques aussi, mais des phénomènes comme les religions et leurs fondamentalismes, la laïcité, les "chocs" dits de cultures, les dégradations environnementales, la «gestion» des risques, la ou les globalisations, les réfugiés, les génocides, les ethnocides, etc., auraient nécessité plus d'attention et de présence écrite de ma part. J'ai peut-être inconsciemment suivi l'exemple de Lévi-Strauss, qui refusait de s'impliquer dans le débat public en France. L'anthropologie est pourtant 


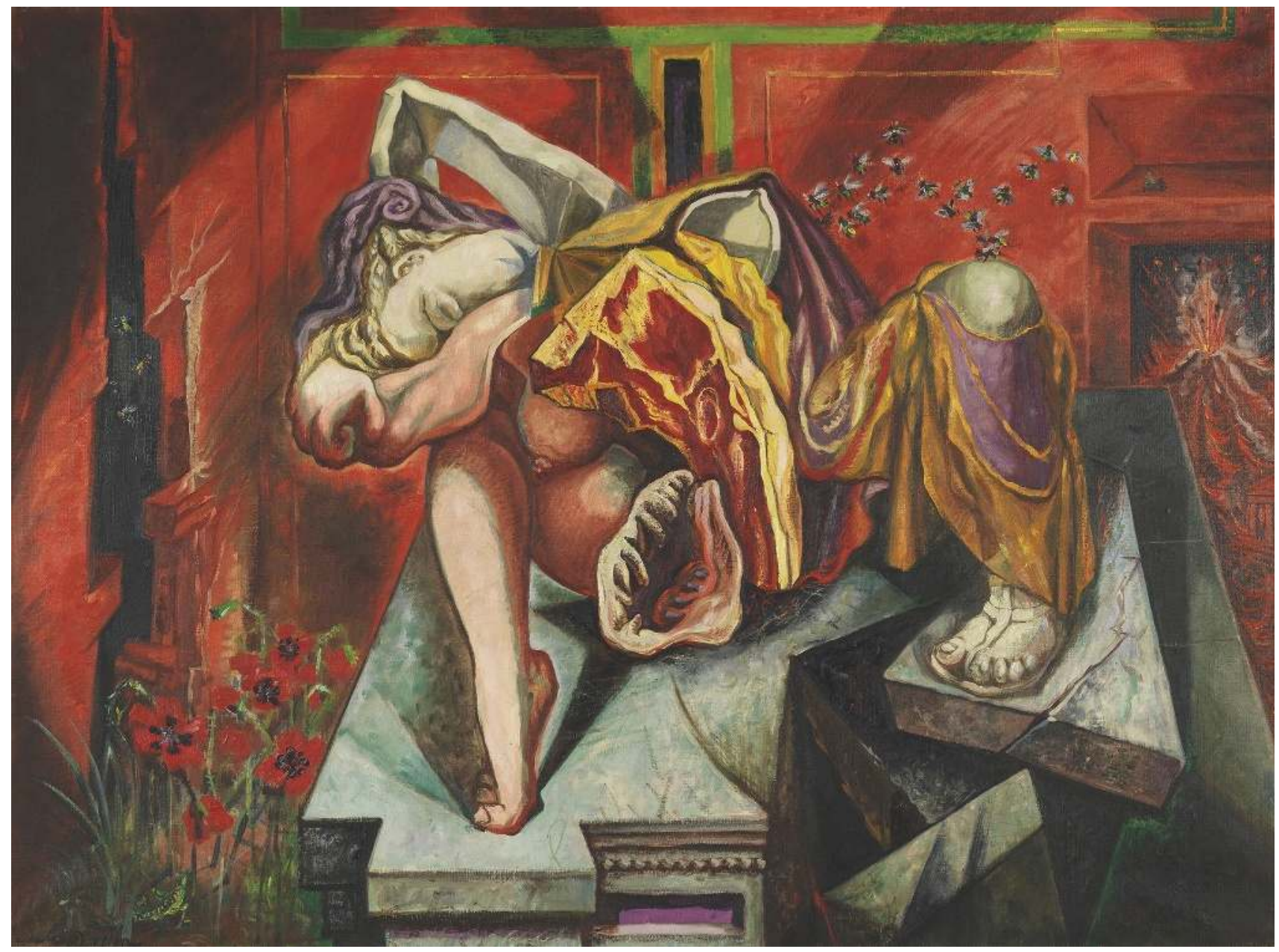

fig. 7

André Masson, Gradiva,

1939, huile sur toile, $97 \times$

$130 \mathrm{~cm}$ ( ) ADAGP, Paris,

2016. Photo @ Centre

Pompidou, MNAM-CCl,

Dist. RMN-Grand Palais /

image Centre Pompidou, MNAM-CCI. 


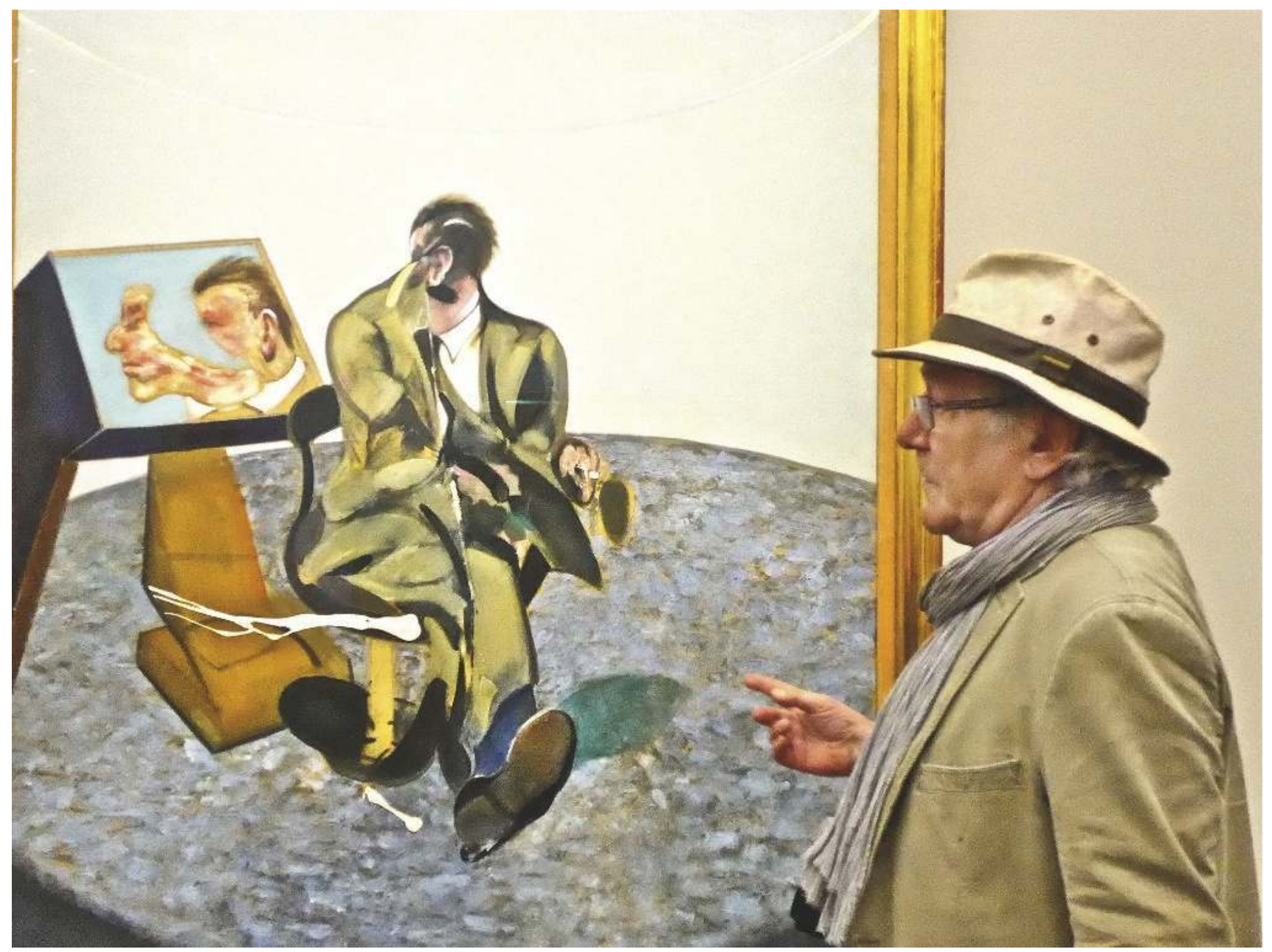

fig. 8

Jean Jamin devant le

Portrait of George Dyer in

a Mirror, par Francis

Bacon, 1968, huile sur

toile, salle Francis Bacon

de l'exposition Leiris \& Co.

centre Pompidou-Metz,

juillet 2015. Cl. Marie-

Élisabeth Handman (C)

Francis Bacon Estate/

ADAGP, Paris 2016. 
une discipline d'observation des phénomènes sociaux et culturels; elle devrait pouvoir intervenir, prendre position, somme toute s'engager comme peuvent le faire et le font encore des anthropologues américains, et comme Leiris lui-même et d'autres l'avaient fait au moment de la guerre d'Algérie, du Congo belge, de Cuba, de la Chine de Mao, du Vietnam, etc. II a existé en France un moment fabuleux, dans les années 1970, où l'anthropologie avait pignon sur rue et faisait l'objet de longues chroniques dans les quotidiens et hebdomadaires; aujourd'hui elle est mise en rayons à côté de rubriques du genre «Ésotérisme», «Mythologie» ou «Développement personnel » dans des grandes surfaces culturelles. Non seulement l'anthropologie est passée de mode, comme est passé de mode le structuralisme qui l'a un moment qualifiée, mais sans doute n'a-t-elle pas su se renouveler ni se donner des objectifs autres que scientifiques, ou prétendument scientifiques, ou a-t-elle versé dans des débats fumeux, abandonnant quelque chose de son âme au profit de théories tout aussi fumeuses empreintes d'un hyper-relativisme désarmant... Je crois cependant - c'est là que vient se nicher peut-être non pas la désillusion biographique mais ma propre illusion biographique - que, dans Gradhiva comme dans L'Homme, j'ai cherché à préserver un pluralisme d'approches, de méthodes, d'idées et de théories, mais que mon pessimisme naturel m'incline à croire révolu: je crains que les chemins de la pensée ne deviennent de moins en moins buissonniers, et ne s'enfoncent dans des certitudes recopiées ou pliées tels des in-quarto. Je n'ai pas hésité non plus - je l'ai déjà signalé -, avec la complicité, entre autres, de plusieurs grands «solistes" de nos formations ethnographiques françaises comme (pour ne citer qu'eux), Françoise Héritier, Jean-Luc Bonniol, Marc Augé, Françoise Zonabend, Patrick Williams, Jean-Pierre Digard, Daniel Fabre - dont la mort laisse pour moi un grand vide ${ }^{\mathbf{3 6}}$-, à proposer des avancées dans les domaines - bien peu politiques et sociétaux certes, mais épistémologiquement séduisants - de l'anthropologie de la littérature, de l'art, de la chanson populaire, du jazz, de la biographie, de l'image (que j'avais esquissées dans Gradhiva) et même de la créolité ou, plus «classiquement », de la parenté et de ses recompositions contemporaines, mais, là encore, sans grande illusion sur la suite qui pourra leur être donnée. Les héritages, les transmissions, ceux et celles que j'ai toujours revendiqués, finissent, avec le temps, par s'étioler ou, pire, par s'oublier, mais sans qu'il soit utile, comme on a trop tendance à le recommander de nos jours, pris par une sorte de fièvre managériale (on n'est pas à un oxymore près!), de les mettre au clou ou d'en faire table rase. Toute retraite n'est-elle pas un chantier d'illusions perdues, une série, comme vous dites, de désillusions? Je ne suis pas loin de m'en convaincre quand il m'arrive de lever le nez et de regarder dans le rétroviseur que me tend notre entretien. D'une certaine manière, il y a un âge où l'on est toujours de la revue!
36. Voir Jean Jamin,

"Daniel Fabre, 1947-2016", L'Homme 217, 2016: 6 ; et Giordana Charuty, Michèle Coquet et Jean Jamin, "In Memoriam...", art. cit.
La mort de Georges Balandier à Paris le 5 octobre 2016 - qui fut l'un des artisans du développement de l'anthropologie en France et l'un de ses penseurs et chroniqueurs avisés, en outre co-inventeur avec Alfred Sauvy du concept de "Tiers-Monde " - a été annoncée au moment où ce numéro 24 de Gradhiva devait partir chez l'imprimeur. Jean Jamin souhaite rappeler ici que c'est sous sa direction qu'il avait entrepris des études de sociologie et d'ethnologie à la Sorbonne, et, à son instigation, qu'il avait réalisé ses premiers travaux d'anthropologie historique sur la "révolte des Mau-Mau » (1952-1957). Grâce à Georges Balandier, Marc Augé et Denise Paulme qui étaient à la tête du comité technique d'anthropologie de l'Orstom (Office de la recherche scientifique et technique outre-mer), Jean Jamin y a été recruté comme élève-chercheur en 1971 et, sous la férule de Pierre Étienne - élève lui aussi de Georges Balandier et de Denise Paulme, condisciple de Marc Augé - a effectué ses premières enquêtes ethnographiques sur les Sénoufo de Côte-d'Ivoire et leur société initiatique, le poro. De cette époque et des discussions qu'il eut avec ces maîtres, depuis devenus des amis, datent son intérêt pour la fonction sociale du secret et ses recherches sur "les lois du silence». Dans les Cahiers internationaux de sociologie, dont il était le directeur, Georges Balandier a publié trois articles importants de Jean Jamin (vol. LXVI-1979, sur l'œuvre de Victor Segalen; vol. LXVII-1979, sur l'anthropologie des Idéologues du Consulat; vol. LXVIII-1980, sur le Collège de sociologie). Georges Balandier s'était aussi lié d'amitié avec Michel Leiris: dans Histoire d'autres (Paris, Stock, 1977), il raconte ses années de formation et sa rencontre avec lui, au musée de l'Homme dans le milieu des années 1940, rencontre qui a joué un rôle essentiel dans ses orientations intellectuelles, scientifiques, et ses engagements moraux et politiques notamment à travers la revue Présence africaine. 